\title{
Analysis of Plume Impingement Effects from Orion Crew Service Module Dual Reaction Control System Engine Firings
}

\author{
A. Prisbell, J. Marichalar \\ Jacobs Technology, Houston, TX 77058, USA \\ and \\ F. Lumpkin, G. LeBeau \\ NASA Johnson Space Center, Houston, TX 77058, USA
}

\begin{abstract}
Plume impingement effects on the Orion Crew Service Module (CSM) were analyzed for various dual Reaction Control System (RCS) engine firings and various configurations of the solar arrays. The study was performed using a decoupled computational fluid dynamics (CFD) and Direct Simulation Monte Carlo (DSMC) approach. This approach included a single jet plume solution for the R1E RCS engine computed with the General Aerodynamic Simulation Program (GASP) CFD code. The CFD solution was used to create an inflow surface for the DSMC solution based on the Bird continuum breakdown parameter. The DSMC solution was then used to model the dual RCS plume impingement effects on the entire CSM geometry with deployed solar arrays. However, because the continuum breakdown parameter of 0.5 could not be achieved due to geometrical constraints and because high resolution in the plume shock interaction region is desired, a focused DSMC simulation modeling only the plumes and the shock interaction region was performed. This high resolution intermediate solution was then used as the inflow to the larger DSMC solution to obtain plume impingement heating, forces, and moments on the CSM and the solar arrays for a total of 21 cases that were analyzed. The results of these simulations were used to populate the Orion CSM Aerothermal Database.
\end{abstract}

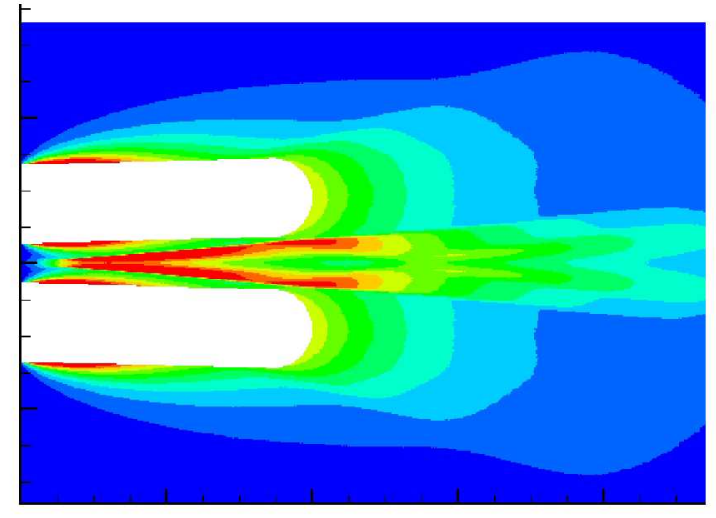

Figure 1: Dual Plume Shock Interaction

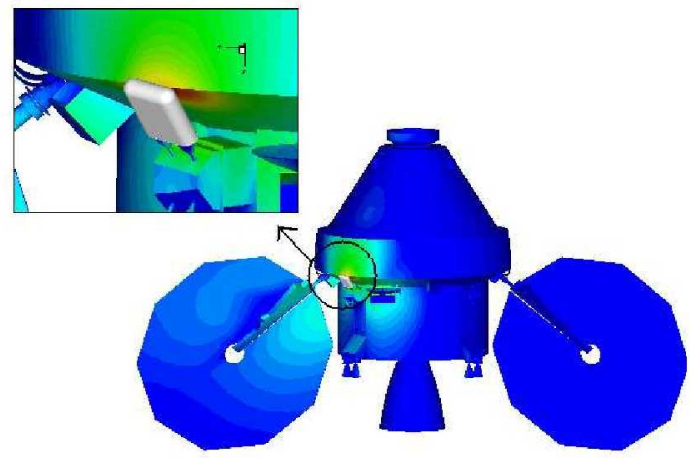

Figure 2: Plume impingement on CSM 


\title{
Analysis of Plume Impingement Effects from Orion Crew Service Module Dual Reaction Control System Engine Firings
}

\author{
A. Prisbell*, J. Marichalar*, F. Lumpkin ${ }^{\dagger}$, G. LeBeau ${ }^{\dagger}$ \\ *Jacobs Technology, Houston, TX 77058, USA \\ ${ }^{\dagger}$ NASA Johnson Space Center, Houston, TX 77058, USA
}

\begin{abstract}
Plume impingement effects on the Orion Crew Service Module (CSM) were analyzed for various dual Reaction Control System (RCS) engine firings and various configurations of the solar arrays. The study was performed using a decoupled computational fluid dynamics (CFD) and Direct Simulation Monte Carlo (DSMC) approach. This approach included a single jet plume solution for the R1E RCS engine computed with the General Aerodynamic Simulation Program (GASP) CFD code. The CFD solution was used to create an inflow surface for the DSMC solution based on the Bird continuum breakdown parameter. The DSMC solution was then used to model the dual RCS plume impingement effects on the entire CSM geometry with deployed solar arrays. However, because the continuum breakdown parameter of 0.05 could not be achieved due to geometrical constraints and because high resolution in the plume shock interaction region is desired, a focused DSMC simulation modeling only the plumes and the shock interaction region was performed. This high resolution intermediate solution was then used as the inflow to the larger DSMC solution to obtain plume impingement heating, forces, and moments on the CSM and the solar arrays for a total of 21 cases that were analyzed. The results of these simulations were used to populate the Orion CSM Aerothermal Database.
\end{abstract}

Keywords: DSMC, Plume Impingement, Rarefied Flow

PACS: 05,51

\section{INTRODUCTION}

The Orion Crew Service Module (CSM) is a part of the Constellation program intended to transport a crew of up to six astronauts into space. The CSM is comprised of two segments - the Crew Module carrying the astronauts and the Service Module serving as the primary propulsion and power component of the system. The CSM Reaction Control System (RCS) is comprised of pairs of axisymmetric R1E thrusters at specific locations on the vehicle and specifically oriented to control the attitude in the pitch, yaw, and roll directions. The RCS thrusters will be used for attitude control in nominal orbit conditions and high altitude abort scenarios. Multiple simultaneous firing combinations of up to eight RCS thrusters are possible. The solar arrays depicted in Fig. 1 are attached to the CSM and will be deployed in a wide range of gimbal angles while in orbit. The plume from the dual RCS thruster firings will impinge upon the CSM and the solar arrays. An understanding of the plume impingement effects produced by the firings of these engines is necessary in formulating an aerothermal environments database for the vehicle.

The close proximity of the engines in the RCS thruster pairings produces complex dual thruster firing plume interaction. The plume interaction region contains shock-shock interactions and amplification heating which is difficult to predict in a vacuum environment with standard Computational Fluid Dynamics (CFD). A CFD solution to adequately capture such complex shock interactions was not available, would take a computationally extended length of time, and would surpass the critical time constraints of this analysis. Moreover, a particle simulation technique such as the Direct Simulation Monte Carlo (DSMC) approach is also computationally expensive in the flow just outside of the nozzle exitplane and in the shock-shock interaction zone due to the extremely high number density of the plume in this region. Therefore, a decoupled CFD/DSMC approach was used. This approach included a single jet firing plume computed with CFD to capture the near exitplane plume characterisitics and two DSMC simulations to capture the shock interaction region and the far flowfield characteristics. One DSMC simulation was used to capture the plume interaction region and another DSMC simulation was used to capture the CSM impingement environment. 


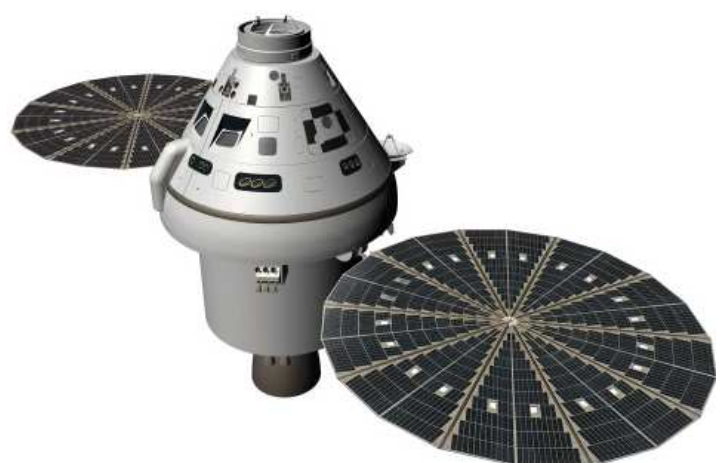

FIGURE 1. Computer representation of CEV with solar arrays extended.

\section{METHODOLOGY}

In the flowfield of an RCS jet, a continuum jet is expelled out of the nozzle and becomes rarefied and eventually reaches the free molecular regime. The Knudsen numbers encompassing the entire nozzle and plume flow field ranges from much less than one to greater than one, making it difficult for DSMC or CFD alone to predict the entire flowfield. A decoupled DSMC/CFD solution is the most common approach [1]. This approach first uses a CFD solution to model the entire plume flowfield. A boundary is determined where the continuum flow breaks down into rarefied flow. This boundary is then used as an input condition for a DSMC solution to model the remainder of the rarefied plume.

The design for the SM calls for using the R1E engine for which CFD simulations already exist. A single engine plume flowfield for the R1E engine was computed in a separate analysis using the General Aerodynamic Simulation Program (GASP) [2]. This CFD analysis was intended to capture the continuum region of the flow. The area where the CFD continuum flow breaks down was determined with the Bird breakdown parameter. The Bird breakdown parameter was computed based on the molecular weight obtained from the mass fractions of the species in the final CFD solution. Figure 2 shows the Bird breakdown parameter computed for the GASP solution with the chosen DSMC inflow boundary location shown as a solid black line. The Bird breakdown parameter in the vicinity of the inflow boundary is in the range of 0.004 to 0.008 , which is far less than the continuum limit of 0.05 [3], and thus the surface lies well within the continuum portion of the flow. This boundary was chosen because of specific geometric constraints including the close proximity of the engines in the RCS thruster pairings and the close proximity of the engine exit plane to the CSM and the solar arrays. The close proximity of the RCS thrusters with each other and the vehicle prevents extraction of a DSMC inflow with a larger Bird breakdown parameter. The distance between the two RCS nozzles is one exit radii and can be seen in Fig. 3. In order to provide an accurate representation of the plume interaction, enough space had to be available for cells to populate the region between the nozzles. This inflow contour did not compromise the validity of the results but merely resulted in a more complex DSMC simulation.

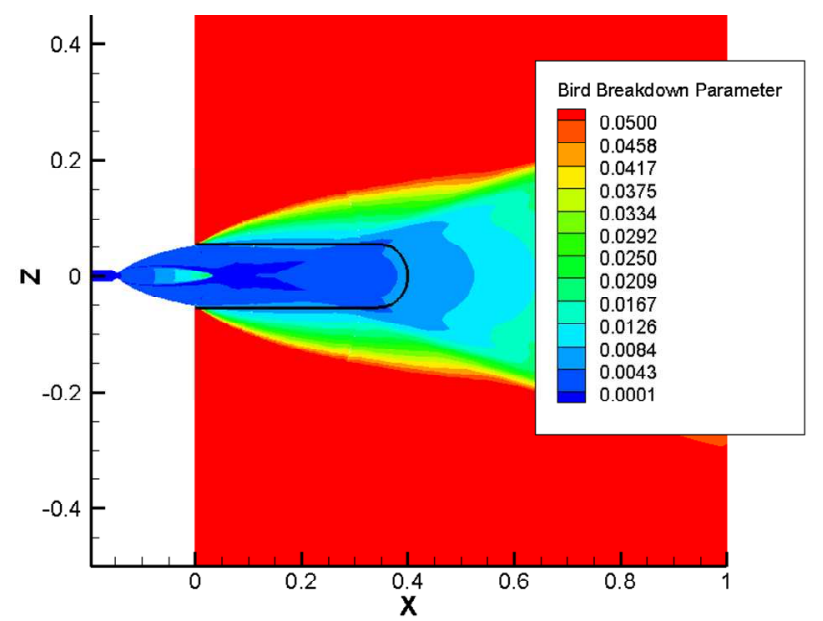

FIGURE 2. CFD solution of continuum flow (DSMC inflow boundary as black line). 


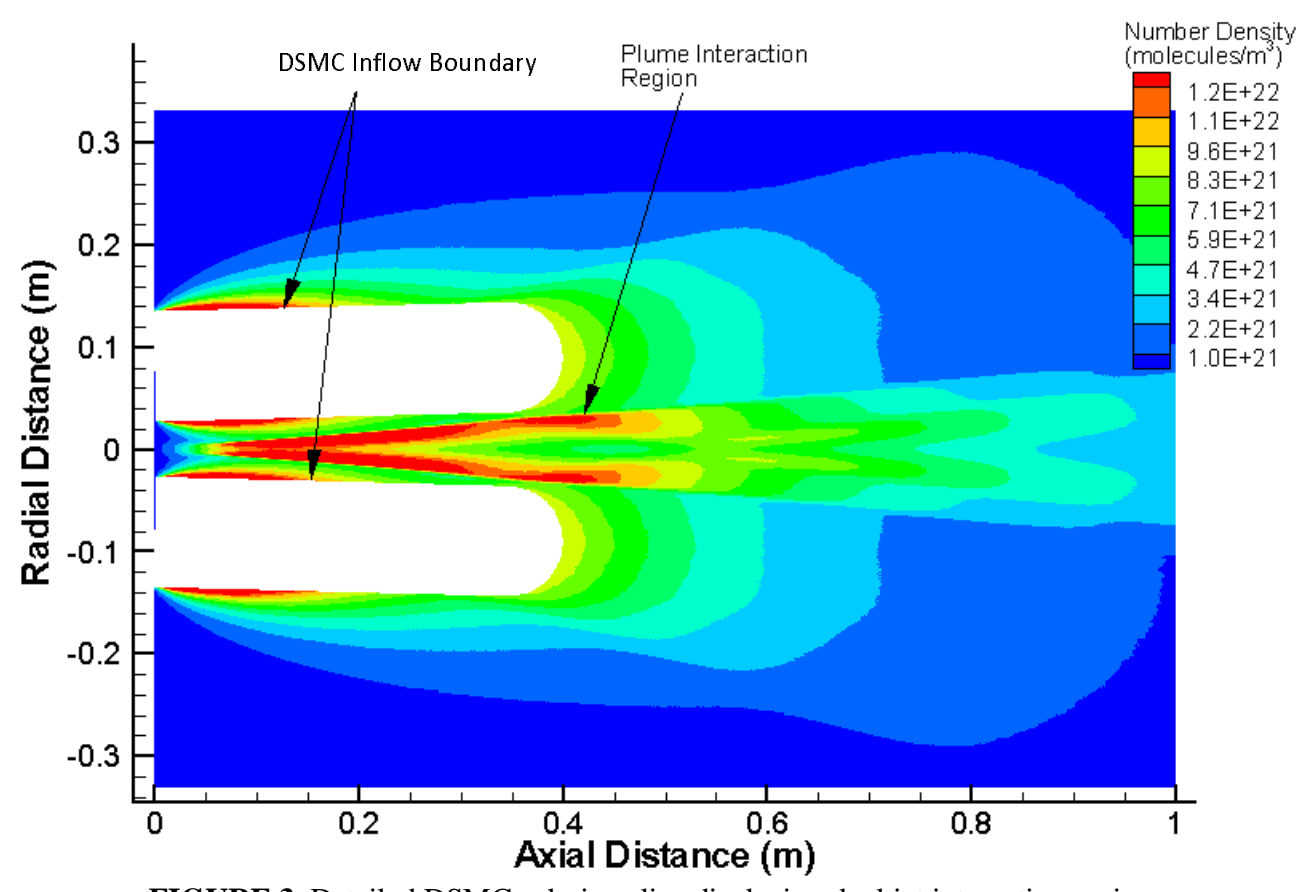

FIGURE 3. Detailed DSMC solution slice displaying dual jet interaction region.

The DSMC simulations were made using the DSMC Analysis Code (DAC) [4]. DAC is the primary program used by NASA Johnson Space Center (JSC) for simulating rarefied flow environments. DAC is a robust code with many utilities. One such utility is the ability to allow varied geometric surfaces to be added to the computational domain which was done for this analysis with the RCS thruster as an inflow boundary. DAC uses a two level Cartesian grid to represent the flowfield cells, with the surface geometry and inflow boundaries embedded within the flowfield grid. The first level is set to a constant and is typically set based on the desired flowfield resolution. The second level of cells is used to refine the flowfield grid in areas of high gradients. The ability of DAC to refine the flowfield grid locally allows DAC to meet the spatial resolution necessary in the area of the plume interaction without excessive refining globally. However, despite this advantage, a detailed, close-up solution of the plume interaction region was necessary to obtain adequate resolution in the shock interaction region between the plumes.

\section{Detailed DSMC Solution}

A high plume number density results when the DSMC inflow boundary has a relatively low Bird breakdown parameter. The close proximity of the two thrusters limited the size and shape of the DSMC inflow boundary that could be used because sufficient separation had to be maintained between the inflows in order to obtain a valid region of shock-shock interaction. A minimum separation distance ensures that flow field interactions do not impinge on the inflow surface and invalidate the applied supersonic inflow boundary condition. It also ensures enough cells are able to be placed in the region between the nozzles to capture the shock-shock interactions with sufficient mean-free-path resolution.

Therefore, in order to capture the plume interaction region accurately, a smaller dimensioned, finer grid had to be used to simulate the flow region just outside the DSMC inflow boundary. A smaller sized computational domain taking advantage of symmetry was created focusing on the inflow boundary, instead of the much larger grid volume necessary to capture the entire CSM surface. A two-dimensional slice of the three-dimensional solution obtained is displayed (mirrored about the Y-axis) in Fig. 3. Despite the efforts to obtain the best quality solution between the plumes, the desired mean-free-path resolution was still not reached in the plume interaction region. Figure 4 depicts the mean-free-path resolution for the plume region. This region has a mean-free-path resolution between 0.1 and 0.2 while the rest of the flowfield is 0.5 and above. A benefit of using DAC in the analysis is the ability to somewhat relax the mean-free-path resolution benchmark of 1.0 for a valid solution based on the nearest neighbor collision methodology that was used with this simulation [5]. The grid was adapted once, and the total amount of simulated molecules reached approximately 500 million. Generally, the minimum cell population in the simulation is ten 
molecules. However, at the edge of the shock interaction cone, the cell population drops below 10 but still remained above 6 in every cell in this area. Given the size of the problem, it is felt that these results are adequate for engineering purposes, especially since no adverse artifacts are apparent.

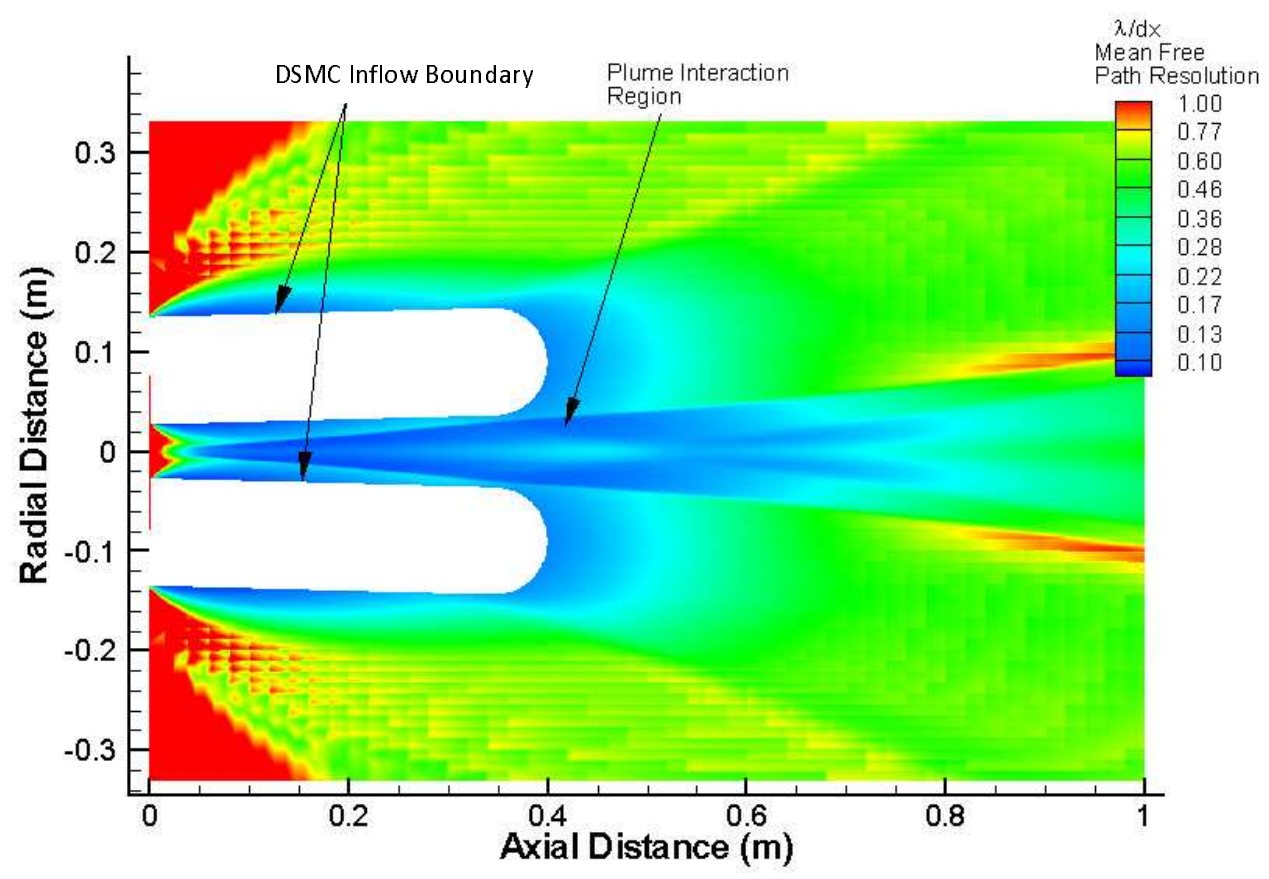

FIGURE 4. Mean-free-path resolution of near field plume region.

\section{Full CSM Solution}

A new inflow surface was extracted from the detailed DSMC solution and is depicted in Fig. 5. This revised inflow decreased the maximum inflow number density and, most importantly, represents an already developed plume interaction region. This greatly enhances the ability to produce valid results when used as an inflow boundary in the much larger computational domain, encompassing the entire CSM and adjacent solar arrays. An average of 20.8 million cells and 200 million simulated molecules existed in the full CSM simulation after the grid was adapted. Each of the 21 simulations had a computational time averaging about 9 hours on 256 processors, equivalent to approximately 2,190 computing hours.

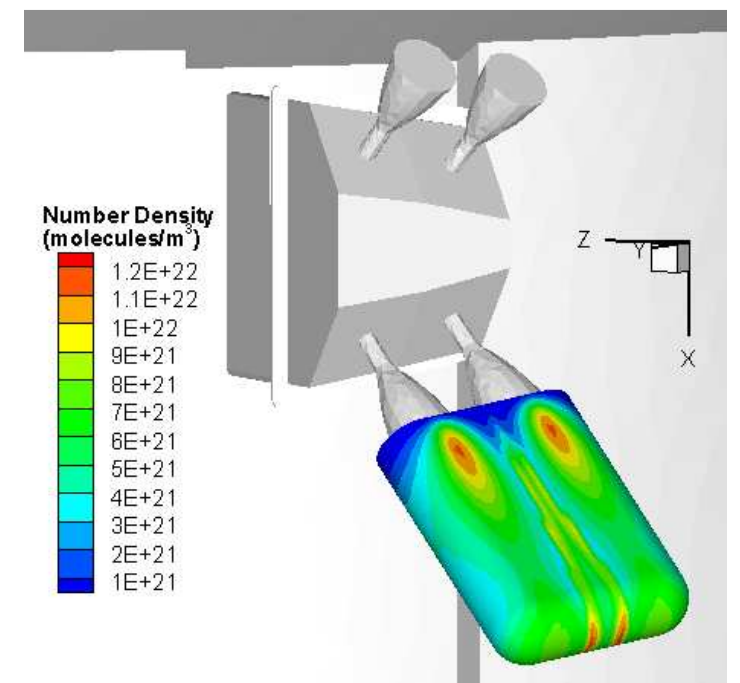

FIGURE 5. DSMC inflow for far flowfield DSMC analysis. 
Figure 6 depicts another geometric constraint that had to be evaluated in order to extract an adequately sized inflow surface for the dual plume inflow boundary. One of the overall goals of this analysis is to obtain the heating rates on the edge of the CSM avionics ring. In order to maintain the validity of the supersonic inflow boundary condition on the inflow surface and obtain sufficient cell resolution between the inflow surface and the CSM avionics ring, enough clearance had to be available between the two zones. This proved to be a challenging task. A smaller inflow surface correlated to a higher number density while a larger inflow surface correlated to decreased mean-free-path resolution in the area of interest and an increased chance of flow field interactions impinging on the inflow surface. A computationally manageable number density inflow was chosen which yielded an average meanfree-path resolution of 0.02 between the inflow surface and the service module geometry. This low value could be the cause of some error in the simulation. An average of about 30 simulated molecules populates the cells in this region.

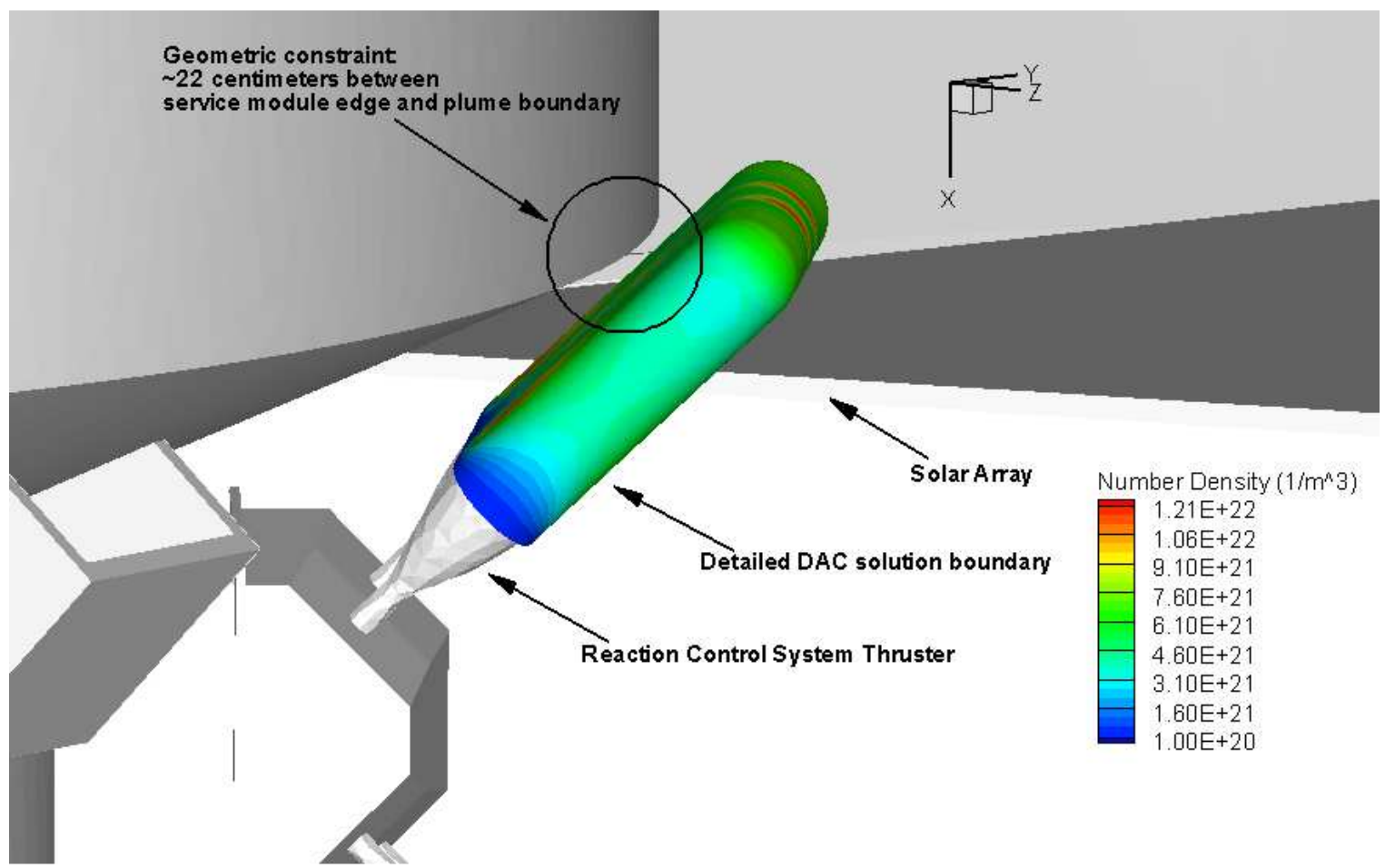

FIGURE 6. Geometric constraints of inflow contour.

\section{SUMMARY OF RESULTS}

A total of 21 cases with varying solar array gimbal angles and varying thruster firing combinations were analyzed using the methodology described above. All of the cases were run conservatively as fully diffuse, and the molecular collision interaction was treated with the nearest neighbor approach [5]. One specie was used to describe the RCS hypergolic plume flowfield. As a result, catalytic energy exchange to the surfaces and chemical energy exchange were nonexistent.

The goal of the analysis was to develop heating data and body forces that are produced by the dual CSM RCS thruster firings. Forces and moments were calculated using DAC post-processing utilities. Figure 7 depicts a solution of one of the 21 cases analyzed. The contours in Fig. 7 represent the total heating on the surface of the vehicle. The impact of the plume wrapping around the surface of the vehicle and engulfing the solar array is evident as expansion occurs in the vacuum environment. The information gathered from this analysis will be used in determining the pressure and heat loads resulting from dual RCS thruster firings for the CSM and will be implemented in the vehicle aerothermal database. 


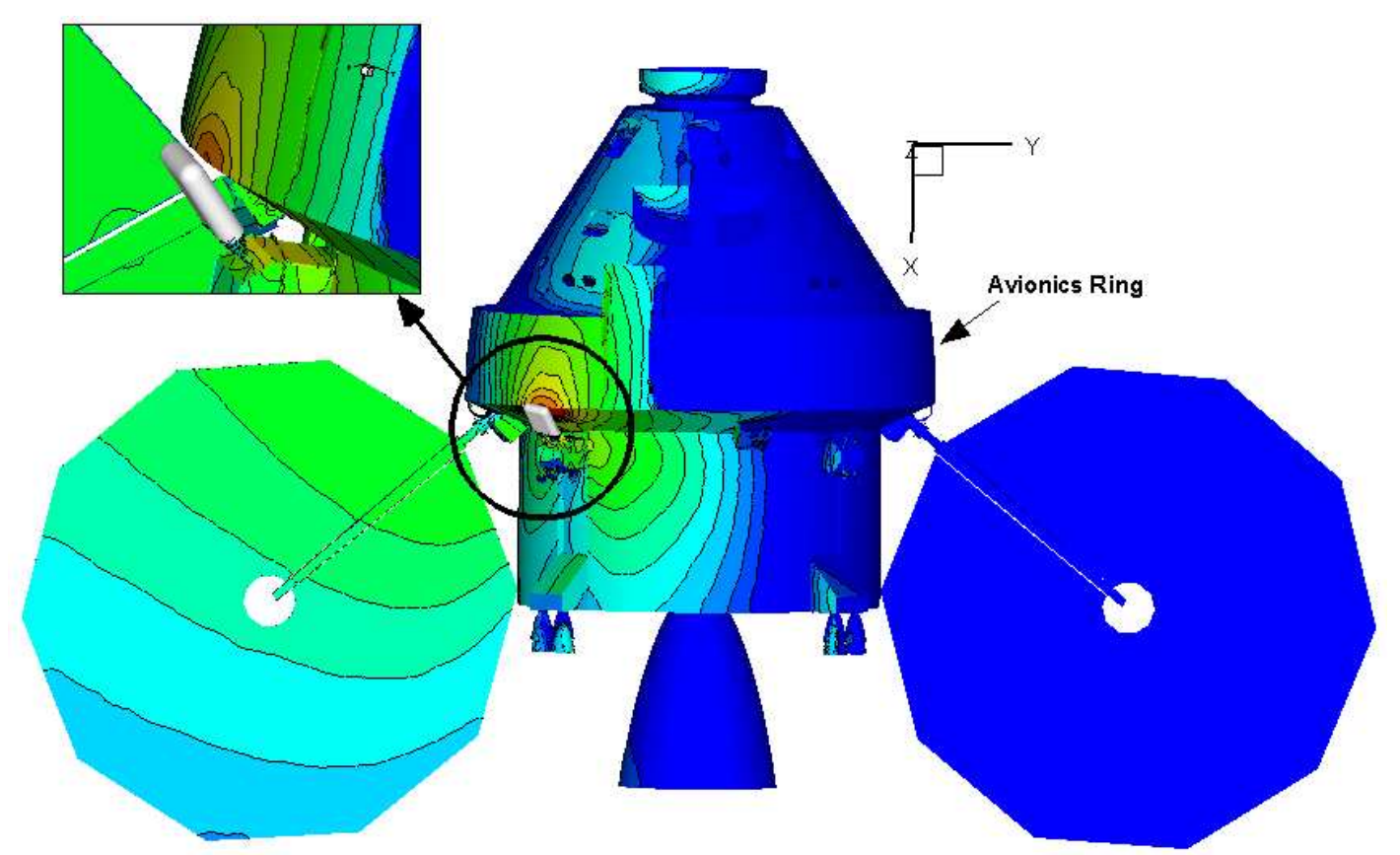

FIGURE 7. Plume Impingement to CSM avionics ring.

\section{REFERENCES}

1. Staack D., McDaniel, J.C., Glass, C. E., Miller, C., "Experimental Study of Interacting Rarefied and Continuum Flows," AIAA 2001-2762, 2001.

2. McGrory, W. D., Stack, D. C., Applebaum, M. P., and Walthers, R. W., "GASP Version 3.1 - The General Aerodynamic Simulation Program,” AeroSoft, Inc., August 1997.

3. Bird, G. A., "Breakdown of Continuum Flow in Free Jets and Rocket Plumes," $12^{\text {th }}$ International Symposium on Rarefied Gas Dynamics, Progress in Aeronautics and Astronautics, 1981.

4. G. J. LeBeau and F. E. Lumpkin III, "Application Highlights of the DSMC Analysis Code (DAC) Software for Simulating Rarefied Flows," Computer Methods in Applied Mechanics and Engineering, 191, No. 6-7, 595-609 (2001).

5. LeBeau, G. J., Boyles, K. A., and Lumpkin, F. E., III, "Virtual Sub-Cells for the Direct Simulation Monte Carlo Method,” AIAA Paper No.2003-1031, 41st AIAA Aerospace Sciences Meeting and Exhibit, Jan. 2003. 